\title{
Effects on Fatty Acid Metabolism of a New Powdered Human Milk Fortifier Containing Medium-Chain Triacylglycerols and Docosahexaenoic Acid in Preterm Infants
}

\author{
Claude Billeaud ${ }^{1}$, Carole Boué-Vaysse ${ }^{2}$, Leslie Couëdelo ${ }^{2}$ (D), Philippe Steenhout ${ }^{3}$, \\ Jonathan Jaeger ${ }^{4}$, Cristina Cruz-Hernandez ${ }^{4}$, Laurent Ameye ${ }^{5}$, Jacques Rigo ${ }^{6}$, \\ Jean-Charles Picaud ${ }^{7}$, Elie Saliba ${ }^{8}$, Nicholas P. Hays ${ }^{5}$ and Frédéric Destaillats ${ }^{5, *}$ \\ 1 CIC Pédiatrique 1401 CHU, 33000 Bordeaux, France; claude.billeaud@chu-bordeaux.fr \\ 2 ITERG, Université de Bordeaux, 33076 Bordeaux, France; c.vaysse@iterg.com (C.B.-V.); \\ 1.couedelo@iterg.com (L.C.) \\ 3 Nestlé Health Sciences, 1066 Epalinges, Switzerland; philippe.steenhout@nestle.com \\ 4 Nestlé Research Centre, 1000 Lausanne, Switzerland; jnthn.jaeger@gmail.com (J.J.); \\ cristina.cruz-hernandez@rdls.nestle.com (C.C.-H.) \\ 5 Nestlé Nutrition R\&D, 1800 Vevey, Switzerland; laurent.ameye@nestle.com (L.A.); \\ nicholaspaul.hays@nestle.com (N.P.H.) \\ 6 Department of Neonatology, University of Liège, 4000 Liège, Belgium; j.rigo@ulg.ac.be \\ 7 Hôpital de la Croix Rousse, Hospices Civils, 69004 Lyon, France; jean-charles.picaud@chu-lyon.fr \\ 8 Hôpital Clocheville, CHU de Tours, 37004 Tours, France; elie.saliba@univ-tours.fr \\ * Correspondence: frederic.destaillats@nestle.com; Tel.: +41-21-924-6217
}

Received: 27 February 2018; Accepted: 25 May 2018; Published: 29 May 2018

\begin{abstract}
Preterm infants require fortification of human milk (HM) with essential fatty acids (FA) to ensure adequate post-natal development. As part of a larger randomized controlled study, we investigated FA metabolism in a subset of 47 clinically stable preterm infants (birth weight $\leq 1500 \mathrm{~g}$ or gestational age $\leq 32$ weeks). Infants were randomized to receive HM supplemented with either a new HM fortifier (nHMF; $n=26$ ) containing $12.5 \mathrm{~g}$ medium-chain FA (MCFA), $958 \mathrm{mg}$ linoleic acid (LA), $417 \mathrm{mg} \alpha$-linolenic acid (ALA), and $157 \mathrm{mg}$ docosahexaenoic acid (DHA) per $100 \mathrm{~g}$ of powder (in compliance with the latest guidelines) or a fat-free HMF (cHMF; $n=21$ ). Plasma phospholipid (PL) and triacylglycerol (TAG), and red blood cell phosphatidylcholine (RBC-PC) and phosphatidylethanolamine (RBC-PE) FA profiles were assessed before and after 21 days of feeding. In the nHMF group, significantly increased levels of n-9 monounsaturated fatty acids were observed, formed most likely by elongation and desaturation of dietary saturated fatty acids present in HM. ALA fortification increased ALA assimilation into plasma TAG. Similarly, DHA fortification enriched the DHA content in RBC-PE, which, in this compartment, was not associated with lower arachidonic acid levels as observed in plasma TAG and phospholipids. RBC-PE, a reliable indicator of FA metabolism and accretion, was the most sensitive compartment in this study.
\end{abstract}

Keywords: arachidonic acid; docosahexaenoic acid; fatty acid metabolism; medium-chain fatty acids; preterm infants

\section{Introduction}

Omega-3 (n-3) and omega-6 (n-6) long-chain polyunsaturated fatty acids (LC-PUFAs) are of crucial importance in early life as they are essential for normal neurodevelopment and growth $[1,2]$. The most biologically active LC-PUFAs are docosahexaenoic acid (DHA; 22:6n-3) and arachidonic 
acid (ARA; 20:4 n-6), which are synthesized from their precursors, $\alpha$-linolenic acid (ALA; 18:3 n-3) and linoleic acid (LA; 18:2 n-6), respectively. Because ALA and LA cannot be synthesized de novo by mammalian cells, they are considered essential fatty acids and therefore must be supplied by the diet. During pregnancy, LC-PUFAs must be derived from maternal sources by placental transfer, and after birth they can only be obtained through the infant diet [3]. LC-PUFAs are essentially accumulated in the central nervous system and other tissues during the third trimester of pregnancy. Infants who are born prematurely are therefore deprived of this rapid in utero accretion and are considered at great risk of LC-PUFA deficiency [1,3,4]. LC-PUFA concentrations in the infant's adipose tissue are insufficient to meet the requirements of the preterm infant and consequently LC-PUFAs required for organ growth are supplied by nutrition, intestinal absorption and conversion from precursor fatty acids [5]. While human milk is considered as the preferred source of nutrition for preterm infants compared to pre-term formula [6], it is also acknowledged that HM provides inadequate protein and micronutrients to support the rapid growth and bone mineralization of preterm infants. HM contains LA, ALA, DHA and ARA; however, the considerable variability of the DHA and ARA content means it may be inadequate for the requirements of preterm infants [5,7]. Consequently, fortification of human milk with essential fatty acids and LC-PUFAs is critical to achieve requirements and to satisfy the considerable demands of growth in the preterm infant $[6,8]$. Combining LC-PUFAs with human-milk fortifiers that increase the calorie, protein and mineral content of human milk has been proposed as a novel approach to supply LC-PUFAs to preterm infants [1]. There are various products used to enrich human milk, including multicomponent fortifiers. To date in Europe, the energy source of multicomponent fortifiers was exclusively carbohydrates. Recently, however, a novel powdered multicomponent fortifier was developed that provides both fats and carbohydrates as energy sources as well as increased levels of protein. The lipid component of this fortifier consists of medium-chain triacylglycerols (MCT) to provide an easily absorbed energy supply, and LC-PUFAs such as DHA, plus additional LA and ALA. We recently demonstrated in a randomized clinical study (http:/ /ClinicalTrials.gov NCT01771588) that this new human milk fortifier (nHMF) is safe, well tolerated, and improves the weight gain of preterm infants, when compared to a control fat-free fortifier (cHMF) [9]. As part of this study, we conducted secondary experiments in a subset of infants to determine the baseline fatty acid composition of unfortified human milk and to compare the effects of nHMF and cHMF on fatty acid metabolism in preterm infants.

\section{Materials and Methods}

\subsection{Study Design and Composition of the Control and New Human Milk Fortifiers}

A randomized, double-blind, multicentre (11 European sites), controlled, parallel-group clinical trial was conducted in clinically stable preterm infants (gestational age $\leq 32$ weeks or birth weight $\leq 1500 \mathrm{~g}$ ) born to mothers who had elected to provide breast milk. Study design, participant characteristics, assessments and objectives have previously been presented in detail [9]. Each subject's parent/legal representative gave their written informed consent for inclusion before participating in the study. The study was conducted in accordance with the Declaration of Helsinki, and the protocol was approved by the Ethics Committee at each study site (initial approval received from Kantonsapotheker Ethikkommission des Kantons Luzern (project identification code EK:1021) on 3 November 2010). Infants tolerating $\geq 100 \mathrm{~mL} / \mathrm{kg} /$ day of human milk for $>24 \mathrm{~h}$ were randomized to receive human milk fortified with cHMF or nHMF until discharge from the neonatal unit or medical decision (minimum 21 days). The composition of the two powdered HMF products is presented in Table 1. To achieve fortification, $5 \mathrm{~g}$ of cHMF or $4 \mathrm{~g}$ of nHMF were added to $100 \mathrm{~mL}$ of human milk. Fortifiers were initially given at half-strength, then increased according to hospital practice, with full-strength fortification occurring once infants were able to ingest $150-180 \mathrm{~mL} / \mathrm{kg} /$ day (i.e., full enteral feeds; study Day 1 ). As described elsewhere [9], sample size was calculated to detect a non-inferior weight gain in infants fed with nHMF versus cHMF from D1 to D21 (non-inferiority margin-1 g/day, expected weight gain 
difference $2 \mathrm{~g}$ /day, standard deviation $4.73 \mathrm{~g}$ /day, type I error 5\%, power 80\%) [9]; 192 subjects (males and females combined) were needed. Group coding was used with two nonspeaking codes per group; fortifier packaging was coded accordingly but otherwise identical in appearance. Infants were enrolled and assigned to their intervention by the study investigators or trained delegates [9]. As part of this study, secondary experiments in a subset of infants were also conducted: in one single centre, fatty acid content was measured in human milk samples, while in three selected centres, lipid status was assessed from blood samples collected on Day 1 and Day 21 of full HM fortification.

Table 1. Nutritional composition of the control (cHMF) and new human milk fortifier (nHMF).

\begin{tabular}{lcc}
\hline \multicolumn{1}{c}{ Nutrients (per 100 g of Powder) * } & cHMF & nHMF \\
\hline Protein (g) & 20.00 & 35.50 \\
Carbohydrates (g) & 66.00 & 32.40 \\
Lipid content (g) $* *$ & 0.38 & 18.10 \\
Saturated fatty acids (g) & - & 12.20 \\
Medium chain fatty acids (MCFA, g) & - & 12.50 \\
Monounsaturated fatty acids (g) & - & 2.93 \\
Linoleic acid (LA, mg) & - & 958.00 \\
(-Linolenic acid (ALA, mg) & - & 417.00 \\
Arachidonic acid (ARA, mg) & - & 13.80 \\
Docosahexaenoic acid (DHA, mg) & - & 157.00 \\
\hline
\end{tabular}

* Vitamins and minerals are not listed. ** Total fatty acid recovery of $0.92 \mathrm{~g}$ per $100 \mathrm{~g}$ of lipids (glycerol and unsaponifiable lipids not measured).

\subsection{Breast Milk and Infant Blood Collection}

In order to characterize the fatty acid content of unfortified and fortified human milk, volunteer mothers of term infants provided breast-milk samples $(n=9)$. Samples were collected after full expression from one breast using a milk pump and while the baby was fed on the other breast. Every effort was made to collect representative samples of a complete feed including fore-, mid-, and hind-milk thus avoiding within-feed variations of lipid and other nutrient levels. Samples were stored at $-80^{\circ} \mathrm{C}$ and shipped on dry ice for analysis at the Nestlé Research Centre, Lausanne, Switzerland. A $25 \mathrm{~mL}$ aliquot was mixed with cHMF (4 g per $100 \mathrm{~mL}$ ) or $\mathrm{nHMF}$ ( $5 \mathrm{~g}$ per $100 \mathrm{~mL}$ ) or analysed as an unfortified sample. Blood samples $(0.7 \mathrm{~mL})$ were collected in EDTA-containing vacutainers from infants during Day 1 and again on Day 21 of full HM fortification. The blood was immediately centrifuged for $10 \mathrm{~min}$ at $1300 \times \mathrm{g}$, and plasma and red blood cells (RBC) were stored in microtubes at $-80{ }^{\circ} \mathrm{C}$ until analysis.

\subsection{Fatty Acid Analysis}

\subsubsection{Breast Milk Fatty Acid Methyl Ester Preparation and Analysis}

Fatty acids were analysed as fatty acid methyl esters (FAMEs) according to the technique recommended by Cruz-Hernandez et al. [10]. The methylation procedure was as follows: $250 \mu \mathrm{L}$ of human milk, then $300 \mu \mathrm{L}$ of internal standard FAME 11:0 and $300 \mu \mathrm{L}$ of internal standard TAG 13:0, $2 \mathrm{~mL}$ of methanol, $2 \mathrm{~mL}$ of methanol/ $\mathrm{HCl}(3 \mathrm{M})$ and $1 \mathrm{~mL}$ of $n$-hexane were added to a $15 \mathrm{~mL}$ test tube with a Teflon-lined screw cap. Test tubes were tightly capped, shaken vigorously, then heated for $60 \mathrm{~min}$ at $100{ }^{\circ} \mathrm{C}$, during which time they were occasionally shaken. Great care was taken to ensure caps were tightly sealed with the cap liner to avoid leaks during heating. The test tubes were cooled to room temperature, then $2 \mathrm{~mL}$ of water were added. The tubes were then shaken vigorously before centrifugation at $1200 \times g$ for $5 \mathrm{~min}$, after which the upper phase ( $n$-hexane) was transferred into gas chromatography (GC) vials. GC analyses were conducted using a 7890 A gas chromatograph with a 7693 autosampler equipped with a preparative station module (Agilent Technologies, Palo Alto, CA, USA) fitted with a fused-silica CP-Sil 88 capillary column (100\% cyanopropylpolysiloxane; $100 \mathrm{~m}$, 
$0.25 \mathrm{~mm}$ ID, $0.25 \mathrm{~mm}$ film thickness; Agilent Technologies, Palo Alto, CA, USA) and a split injector (1:25 ratio) heated to $250{ }^{\circ} \mathrm{C}$ and a flame ionization detector operated at $300{ }^{\circ} \mathrm{C}$. The oven temperature was programmed at $60{ }^{\circ} \mathrm{C}$ isothermal for $5 \mathrm{~min}$, increased by $15^{\circ} \mathrm{C} / \mathrm{min}$ to $165^{\circ} \mathrm{C}$, isothermal for $1 \mathrm{~min}$ at this temperature, then increased to $195^{\circ} \mathrm{C}$ by $2{ }^{\circ} \mathrm{C} / \mathrm{min}$ and held isothermal for $14 \mathrm{~min}$, and then increased to $215^{\circ} \mathrm{C}$ by $5^{\circ} \mathrm{C} / \mathrm{min}$ and held isothermal for $8 \mathrm{~min}$ at $215^{\circ} \mathrm{C}$. Hydrogen was used as carrier gas in constant flow mode at $1.5 \mathrm{~mL} / \mathrm{min}$.

\subsubsection{Plasma Lipid Class Separation}

Lipids were extracted from plasma according to the technique recommended by Folch et al. [11]. Lipid classes were separated by thin-layer chromatography (TLC) and sample migration was performed with hexane/diethyl ether/acetic acid $(80 / 20 / 1 ; v / v / v)$. After drying, the lipid classes were visualized by spraying the TLC plate with 1,2-dichlorofluorescein and detecting under UV-light. The lipid fractions (PL and TAG) were identified by comparison with standards and collected in glass tubes. Standard TAG 17:0 and PE 17:0 were added to the TAG and PL extracts, respectively.

\subsubsection{RBC Phospholipid Class Separation}

Lipids were extracted from the RBC according to the method recommended by Peuchant [12]. PC and PE were separated from the RBC lipid extract by TLC and sample migration was performed with chloroform/methanol/acetic acid/water (50/37.5/3.5/2; v/v/v). PC and PE were visualized by spraying the TLC with 1,2-dichlorofluorescein and detecting under UV-light. The lipid fractions from PC and PE were identified by comparison with standards then collected in glass tubes. Standard PE and PC 17:0 were added to the PE and PC extracts, respectively.

\subsubsection{Plasma and RBC FAME Preparation and Analysis}

Fatty acids in plasma TAG and PL were transesterified according to the method described by Morrison and Smith [13]. FAMEs were analysed by GC on a BPX 70 capillary column (60 m long, $0.25 \mu \mathrm{m}$ film, $0.25 \mathrm{~mm}$ ID, SGE, Milton Keynes, UK). Hydrogen was used as carrier gas with a constant $120 \mathrm{kPa}$ pressure and flow of $20 \mathrm{~mL} / \mathrm{min}$. The GC system consisted of a Focus GS (Thermofinnigan, Courtaboeuf, France) equipped with a split injector (1:80 ratio) heated to $250^{\circ} \mathrm{C}$ and a flame ionization detector operated at $250^{\circ} \mathrm{C}$. The column temperature was increased from $150{ }^{\circ} \mathrm{C}$ to $200{ }^{\circ} \mathrm{C}\left(1.3^{\circ} \mathrm{C} / \mathrm{min}\right)$, maintained at $200{ }^{\circ} \mathrm{C}$ for $20 \mathrm{~min}$, increased from $200{ }^{\circ} \mathrm{C}$ to $235^{\circ} \mathrm{C}\left(10^{\circ} \mathrm{C} / \mathrm{min}\right)$, and held at $235^{\circ} \mathrm{C}$ for $20 \mathrm{~min}$. ChromQuest software (Thermofinnigan, Courtaboeuf, France) was used for data acquisition and handling. A pure FAME mixture (Sigma, St Louis, MO, USA) of known composition was used as the standard for column calibration. The variation in peak area between injections was below $2 \%$.

\subsection{Statistical Analysis}

Levels of fatty acids were measured at Day 1 and Day 21 of full HM fortification, in plasma PL, plasma TAG, RBC-PC and RBC-PE. Summary statistics for each fatty acid and for each compartment were calculated at each visit. Data distribution was close to log-normal; geometric mean and geometric standard deviation are provided in the present paper instead of arithmetic mean and standard deviation. Relative fatty acid concentrations were analysed at Day 21 (log-transformation) using a mixed-effect ANCOVA model adjusted for postmenstrual age at Day 1, weight at Day 1, fatty acid concentration at Day 1, sex, centre and treatment group (with centre considered as a random effect). Estimations of the treatment effect $\mathrm{nHMF} / \mathrm{cHMF}$ (log of the ratio of the geometric estimates) and the two-sided $p$-value are given in for each fatty acid analysed in the different lipid compartments. 


\section{Results}

\subsection{Study Population}

As reported elsewhere [9], 153 premature infants were enrolled in this clinical study and randomized to receive $\operatorname{nHMF}(n=77)$ or $\mathrm{cHMF}(n=76)$. Infant demographic and baseline anthropometric characteristics and the number of twins were similar in both groups [9]. Fatty acid profiles in plasma PL and TAG, RBC-PC and RBC-PE were analysed in a subset of 47 infants $(n=21$ and $n=26$ fed with cHMF and $\mathrm{nHMF}$, respectively).

\subsection{Fatty Acids in Human Milk}

The quantitative fatty acid profile of nine samples of human milk was determined. Table 2 shows the fatty acid composition (mg per $100 \mathrm{~mL}$ ) of unfortified human milk and of human milk fortified with cHMF or nHMF. Of the medium-chain FA measured, 8:0 and 10:0 were increased in human milk fortified with $\mathrm{nHMF}$ when compared to $\mathrm{CHMF}$ due to the use of medium-chain triglyceride oil in the nHMF. LA, ALA, EPA and DHA levels were also greater (nearly twice) in the nHMF than in the cHMF. According to the estimated levels of essential and LC-PUFAs provided by human milk fortified with cHMF or nHMF in preterm infants (Table 3), human milk fortified with nHMF provided the recommended intakes of essential fatty acids and LC-PUFAs for premature infants $[6,14,15]$. It is important to note that the limited number of samples $(n=9)$ does not allow estimation of the variable composition of the fortified HM, resulting from natural fluctuation of macronutrient content and composition (i.e., lipids), or impact on processing steps such as HM collection, handling and pasteurization.

Table 2. Fatty acid content (mg/100 mL) of unfortified human milk * and human milk fortified with a control (cHMF) or a new human milk fortifier (nHMF), and level (\%) of fortification achieved with nHMF relative to $\mathrm{CHMF}$.

\begin{tabular}{|c|c|c|c|c|c|c|c|}
\hline \multirow{2}{*}{ Fatty Acid } & \multicolumn{2}{|c|}{ Human Milk (HM, $n=9$ ) } & \multicolumn{2}{|c|}{ HM Fortified with cHMF $(n=9)$} & \multicolumn{2}{|c|}{ HM Fortified with nHMF $(n=9)$} & \multirow{2}{*}{$\begin{array}{c}\begin{array}{c}\text { Fortification } * * \\
\text { (nHMF vs. cHMF }\end{array} \\
\%\end{array}$} \\
\hline & Mean & SD & Mean & SD & Mean & SD & \\
\hline $6: 0$ & 2.1 & 0.9 & 2.1 & 1.2 & 2.2 & 1.4 & - \\
\hline $8: 0$ & 8.3 & 2.5 & 10.5 & 3.4 & 243.8 & 17.5 & 2222 \\
\hline 10:0 & 55.3 & 18.5 & 56.4 & 22.5 & 245.2 & 24.9 & 335 \\
\hline $12: 0$ & 190.1 & 62.3 & 191.6 & 81.5 & 190.2 & 80.1 & - \\
\hline $14: 0$ & 233.3 & 64.2 & 236.9 & 95.9 & 242.2 & 100.2 & - \\
\hline $16: 0$ & 768.0 & 227.8 & 771.3 & 274.1 & 737.0 & 272.3 & - \\
\hline $16: 1 n-7$ & 71.0 & 30.2 & 68.8 & 29.9 & 93.5 & 101.1 & 36 \\
\hline 18:0 & 212.7 & 58.9 & 213.7 & 69.0 & 204.5 & 79.5 & - \\
\hline trans-18:1 & 25.0 & 11.2 & 24.7 & 13.0 & 32.3 & 33.8 & 31 \\
\hline $18: 1 n-9$ & 1096.9 & 275.4 & 1079.7 & 277.9 & 1149.6 & 272.4 & - \\
\hline $18: 1 n-7$ & 65.6 & 15.3 & 65.5 & 22.7 & 71.6 & 20.8 & - \\
\hline $\begin{array}{c}18: 2 n-6 \\
\text { (LA) }\end{array}$ & 317.6 & 105.7 & 311.1 & 92.3 & 336.2 & 92.5 & - \\
\hline $\begin{array}{c}18: 3 n-3 \\
\text { (ALA) }\end{array}$ & 29.5 & 10.7 & 28.5 & 8.8 & 43.8 & 10.2 & 54 \\
\hline $\begin{array}{c}18: 3 n-6 \\
\text { (GLA) }\end{array}$ & 2.6 & 1.4 & 2.7 & 1.6 & 2.6 & 1.3 & - \\
\hline 20:0 & 6.0 & 2.2 & 6.2 & 2.4 & 6.8 & 2.2 & 10 \\
\hline $20: 1 n-9$ & 15.7 & 4.7 & 15.7 & 5.3 & 17.4 & 5.6 & 11 \\
\hline $20: 2 n-6$ & 9.8 & 3.8 & 10.1 & 4.1 & 10.0 & 4.0 & - \\
\hline $\begin{array}{l}20: 3 n-6 \\
\text { (DGLA) }\end{array}$ & 13.3 & 6.3 & 13.5 & 8.4 & 13.0 & 6.9 & - \\
\hline $22: 1 n-9$ & 2.9 & 1.2 & 3.1 & 1.4 & 3.0 & 1.5 & - \\
\hline $\begin{array}{c}20: 4 n-6 \\
\text { (ARA) }\end{array}$ & 15.9 & 7.3 & 15.7 & 9.1 & 15.8 & 8.0 & - \\
\hline $\begin{array}{c}20: 5 n-3 \\
\text { (EPA) }\end{array}$ & 2.3 & 1.1 & 2.1 & 0.9 & 3.4 & 1.2 & 62 \\
\hline $24: 0$ & 3.2 & 1.3 & 3.4 & 1.7 & 3.6 & 1.6 & - \\
\hline $24: 1 n-9$ & 3.5 & 1.9 & 3.6 & 2.4 & 3.9 & 2.5 & - \\
\hline $\begin{array}{c}22: 6 n-3 \\
\text { (DHA) }\end{array}$ & 12.7 & 5.8 & 12.5 & 6.3 & 18.1 & 6.7 & 45 \\
\hline
\end{tabular}

${ }^{*}$ Data based on analysis of 9 donor milk samples. ${ }^{* *}$ Values reported $\geq 10 \%$. ALA, $\alpha$-linolenic acid; ARA, arachidonic acid; DGLA, dihomo- $\gamma$-linolenic acid; DHA, docosahexaenoic acid; EPA, eicosapentaenoic acid; GLA, $\gamma$-linolenic acid; LA, linoleic acid; SD, standard deviation. 
Table 3. Estimated levels of essential and long-chain polyunsaturated fatty acids provided by human milk (HM) fortified with a control (cHMF) or a new human milk fortifier (nHMF) in preterm infant study participants $\left(\mathrm{mg} \cdot \mathrm{kg}^{-1} \cdot \mathrm{day}^{-1}\right)$. Comparison with recent expert panel recommendations [6,14,15].

\begin{tabular}{ccccccc}
\hline & & HM * Fortified with & \multicolumn{3}{c}{ Recent Recommendations } \\
\cline { 3 - 7 } Fatty Acid & Unit & cHMF & nHMF & $\begin{array}{c}\text { ESPGHAN } \\
\text { 2010 [15] }\end{array}$ & $\begin{array}{c}\text { Lapillonne et al. } \\
\text { 2014 [6] }\end{array}$ & $\begin{array}{c}\text { Koletzko et al. } \\
\text { 2014 [14] }\end{array}$ \\
\hline 18:2 $n-6$ (LA) & $\mathrm{mg} \cdot \mathrm{kg}^{-1} \cdot \mathrm{day}^{-1}$ & 475 & 513 & $350-1400$ & $350-1400$ & $385-1540$ \\
18:3 $n$-3 (ALA) & $\mathrm{mg} \cdot \mathrm{kg}^{-1} \cdot \mathrm{day}^{-1}$ & 44 & 67 & $>55$ & $>55$ & $>55$ \\
LA:ALA & - & $11: 1$ & $8: 1$ & $5-15: 1$ & $5-15: 1$ & - \\
20:4 $n$-6 (ARA) & $\mathrm{mg} \cdot \mathrm{kg}^{-1} \cdot \mathrm{day}^{-1}$ & 24 & 24 & $18-42$ & $18-45$ & $18-45$ \\
22:6 $n$-3 (DHA) & $\mathrm{mg} \cdot \mathrm{kg}^{-1} \cdot \mathrm{day}^{-1}$ & 19 & 28 & $12-30$ & $12-60$ & $18-60$ \\
ARA:DHA & - & $1.3: 1$ & $0.8: 1$ & $1-2: 1$ & - & - \\
20:5 $n$-3 (EPA) & $\mathrm{mg} \cdot \mathrm{kg}^{-1} \cdot \mathrm{day}^{-1}$ & $3^{* *}$ & $5^{+}$ & $<30 \%$ DHA & $<20$ & $<20$ \\
\hline
\end{tabular}

* Estimation based on 9 donor human milk samples collected in the present study. ${ }^{* *} 16 \%$ of DHA supply. ${ }^{\dagger} 18 \%$ of DHA supply. ALA, $\alpha$-linolenic acid; ARA, arachidonic acid; DHA, docosahexaenoic acid; EPA, eicosapentaenoic acid; LA, linoleic acid.

\subsection{Fatty Acid Profile in Plasma and RBC Lipids of Preterm Infants Fed cHMF or nHMF}

The fatty acid profile (expressed as g per $100 \mathrm{~g}$ of fatty acids) of plasma PL, plasma TAG, RBC-PC and RBC-PE fractions in the two study groups (nHMF and CHMF), before and after 21 days of feeding, are reported in Tables $4-7$ respectively.

Table 4. Fatty acid profile (g/100 g of fatty acids) of total plasma phospholipids in preterm infants, receiving human milk fortified with a control (cHMF) or with a new human milk fortifier (nHMF) before and after 21 days of treatment. Estimates of the treatment effect nHMF/cHMF (difference) and the two-sided $p$-values are given for each fatty acid analyzed in the different lipid compartments.

\begin{tabular}{|c|c|c|c|c|c|c|c|c|c|c|}
\hline & \multicolumn{4}{|c|}{ cHMF $(n=21)$} & \multicolumn{4}{|c|}{ nHMF $(n=26)$} & \multirow{3}{*}{ Difference } & \multirow{3}{*}{$p$ Value } \\
\hline & \multicolumn{2}{|c|}{ Baseline } & \multicolumn{2}{|c|}{ After 21 Days } & \multicolumn{2}{|c|}{ Baseline } & \multicolumn{2}{|c|}{ After 21 Days } & & \\
\hline & Mean & SD & Mean & SD & Mean & SD & Mean & SD & & \\
\hline $14: 0$ & 0.21 & 0.14 & 0.22 & 0.14 & 0.24 & 0.13 & 0.26 & 0.13 & 0.184 & 0.500 \\
\hline $15: 0$ & 0.13 & 0.10 & 0.18 & 0.26 & 0.14 & 0.08 & 0.12 & 0.03 & -0.071 & 0.722 \\
\hline 16:0 & 25.00 & 5.55 & 22.75 & 6.21 & 26.00 & 3.58 & 26.46 & 2.76 & 0.180 & 0.020 \\
\hline 16:0 DMA & 0.63 & 0.18 & 0.70 & 0.17 & 0.64 & 0.16 & 0.74 & 0.27 & -0.134 & 0.394 \\
\hline $16: 1 n-7$ & 1.24 & 0.71 & 1.05 & 1.19 & 1.28 & 0.69 & 0.93 & 0.70 & 0.209 & 0.188 \\
\hline $16: 1 n-9$ & 0.32 & 0.14 & 0.24 & 0.15 & 0.30 & 0.12 & 0.25 & 0.13 & 0.219 & 0.117 \\
\hline 18:0 & 14.99 & 2.90 & 17.25 & 4.15 & 14.51 & 1.65 & 15.84 & 1.63 & -0.060 & 0.203 \\
\hline 18:0 DMA & 0.33 & 0.12 & 0.47 & 0.37 & 0.35 & 0.10 & 0.36 & 0.14 & -0.402 & 0.040 \\
\hline 18:1 DMA & 0.29 & 0.14 & 0.32 & 0.11 & 0.30 & 0.09 & 0.30 & 0.13 & -0.288 & 0.054 \\
\hline $18: 1 n-7$ & 3.07 & 0.59 & 2.36 & 0.62 & 3.05 & $0 ; 89$ & 2.59 & 0.77 & 0.122 & 0.015 \\
\hline $18: 1 n-9$ & 14.04 & 2.16 & 11.98 & 3.37 & 13.90 & 3.03 & 12.45 & 2.97 & 0.051 & 0.286 \\
\hline trans-18:1 & 0.30 & 0.15 & 0.42 & 0.16 & 0.39 & 0.13 & 0.40 & 0.17 & -0.112 & 0.515 \\
\hline $18: 2$ n-6 (LA) & 12.90 & 3.20 & 14.60 & 2.53 & 14.03 & 2.75 & 14.69 & 2.55 & 0.043 & 0.452 \\
\hline 18:3n-3 (ALA) & 0.18 & 0.14 & 0.11 & 0.05 & 0.18 & 0.10 & 0.17 & 0.08 & 0.219 & 0.125 \\
\hline 18:3 n-6 (GLA) & 0.22 & 0.07 & 0.17 & 0.06 & 0.22 & 0.06 & 0.16 & 0.04 & 0.030 & 0.689 \\
\hline 20:0 & 0.36 & 0.29 & 0.40 & 0.31 & 0.38 & 0.21 & 0.41 & 0.18 & 0.011 & 0.945 \\
\hline $20: 1 n-9$ & 0.25 & 0.08 & 0.31 & 0.09 & 0.27 & 0.10 & 0.36 & 0.12 & 0.156 & 0.072 \\
\hline $20: 2 n-6$ & 0.47 & 0.13 & 0.67 & 0.46 & 0.51 & 0.11 & 0.53 & 0.10 & -0.112 & 0.340 \\
\hline $20: 3 n-6$ (DGLA) & 3.79 & 0.89 & 4.04 & 1.05 & 3.46 & 0.72 & 3.45 & 0.68 & -0.161 & 0.041 \\
\hline $20: 3 n-9$ & 2.56 & 1.46 & 1.90 & 1.53 & 2.12 & 1.19 & 2.11 & 1.51 & 0.089 & 0.612 \\
\hline $20: 4 n-6$ (ARA) & 9.81 & 2.33 & 10.63 & 3.54 & 9.09 & 2.03 & 8.26 & 2.58 & -0.211 & 0.015 \\
\hline $20: 5 n-3$ (EPA) & 0.84 & 0.48 & 0.70 & 0.25 & 0.88 & 0.32 & 0.97 & 0.28 & 0.318 & 0.006 \\
\hline $22: 0$ & 0.46 & 0.39 & 0.53 & 0.53 & 0.53 & 0.33 & 0.57 & 0.27 & 0.110 & 0.567 \\
\hline $22: 1 n-9$ & 0.05 & 0.03 & 0.05 & 0.03 & 0.05 & 0.02 & 0.05 & 0.02 & 0.048 & 0.676 \\
\hline $22: 4 n-6$ & 0.46 & 0.17 & 0.49 & 0.29 & 0.40 & 0.13 & 0.35 & 0.10 & -0.257 & 0.032 \\
\hline $22: 5 n-3(n-3$ DPA $)$ & 0.46 & 0.24 & 0.53 & 0.31 & 0.44 & 0.12 & 0.40 & 0.12 & -0.217 & 0.037 \\
\hline $22: 5 n-6(n-6$ DPA $)$ & 0.49 & 0.19 & 0.53 & 0.25 & 0.45 & 0.25 & 0.50 & 0.22 & -0.005 & 0.970 \\
\hline $22: 6 n-3$ (DHA) & 3.60 & 1.42 & 3.96 & 1.94 & 3.22 & 0.93 & 3.54 & 1.04 & -0.050 & 0.575 \\
\hline $24: 0$ & 0.39 & 0.29 & 0.53 & 0.80 & 0.48 & 0.28 & 0.43 & 0.18 & -0.056 & 0.742 \\
\hline $24: 1 n-9$ & 1.50 & 1.23 & 1.47 & 0.83 & 1.55 & 0.98 & 1.77 & 0.73 & 0.191 & 0.368 \\
\hline
\end{tabular}

Data are presented as geometric mean and geometric standard deviation (SD). ALA, $\alpha$-linolenic acid; ARA, arachidonic acid; DGLA, dihomo- $\gamma$-linolenic acid; DHA, docosahexaenoic acid; DMA, dimethyl acetal; DPA, docosapentaenoic acid; EPA, eicosapentaenoic acid; GLA, $\gamma$-linolenic acid; LA, linoleic acid. 
Table 5. Fatty acid profile (g/100 g of fatty acids) of plasma triacylglycerols in preterm infants receiving human milk fortified with a control (cHMF) or with a new human milk fortifier (nHMF), before and after 21 days of treatment. Estimations of the treatment effect nHMF/cHMF (Difference) and the two-sided $p$-value are given for each fatty acid analysed in the different lipid compartments.

\begin{tabular}{|c|c|c|c|c|c|c|c|c|c|c|}
\hline \multirow{2}{*}{ Fatty Acid } & \multicolumn{4}{|c|}{ cHMF $(n=21)$} & \multicolumn{4}{|c|}{ nHMF $(n=26)$} & \multirow{2}{*}{ Difference } & \multirow{2}{*}{$p$ Value } \\
\hline & Mean & SD & Mean & SD & Mean & SD & Mean & SD & & \\
\hline 14:0 & 2.23 & 1.09 & 2.22 & 1.38 & 2.77 & 1.40 & 2.56 & 1.36 & 0.243 & 0.262 \\
\hline iso-16:0 & 0.05 & 0.02 & 0.05 & 0.02 & 0.06 & 0.02 & 0.06 & 0.02 & 0.203 & 0.259 \\
\hline 15:0 & 0.26 & 0.12 & 0.27 & 0.06 & 0.27 & 0.07 & 0.28 & 0.08 & -0.013 & 0.868 \\
\hline $16: 1 n-9$ & 0.96 & 0.26 & 0.87 & 0.24 & 0.95 & 0.23 & 0.93 & 0.26 & 0.087 & 0.226 \\
\hline $17: 1$ & 0.25 & 0.04 & 0.23 & 0.04 & 0.24 & 0.04 & 0.25 & 0.06 & 0.078 & 0.176 \\
\hline 18:0 & 3.73 & 0.79 & 4.68 & 0.84 & 4.22 & 1.17 & 4.77 & 0.74 & -0.032 & 0.529 \\
\hline $18: 1 n-7$ & 4.71 & 1.39 & 4.12 & 1.56 & 4.31 & 1.35 & 4.32 & 1.66 & 0.065 & 0.371 \\
\hline $18: 1 n-9$ & 43.18 & 2.50 & 40.22 & 3.91 & 39.68 & 4.39 & 38.41 & 3.33 & -0.018 & 0.517 \\
\hline trans-18:1 & 0.35 & 0.13 & 0.53 & 0.16 & 0.47 & 0.21 & 0.42 & 0.15 & -0.289 & 0.012 \\
\hline $20: 0$ & 0.11 & 0.03 & 0.13 & 0.05 & 0.11 & 0.04 & 0.13 & 0.04 & -0.008 & 0.941 \\
\hline $20: 1 n-7$ & 0.14 & 0.05 & 0.16 & 0.07 & 0.13 & 0.05 & 0.13 & 0.05 & -0.105 & 0.363 \\
\hline $20: 1 n-9$ & 0.47 & 0.07 & 0.51 & 0.09 & 0.49 & 0.10 & 0.54 & 0.07 & 0.069 & 0.042 \\
\hline $20: 2 n-6$ & 0.18 & 0.08 & 0.22 & 0.09 & 0.20 & 0.07 & 0.19 & 0.10 & -0.147 & 0.232 \\
\hline 20:3 n-6 (DGLA) & 0.27 & 0.08 & 0.32 & 0.13 & 0.25 & 0.11 & 0.25 & 0.15 & -0.221 & 0.075 \\
\hline $20: 3 n-9$ & 0.72 & 0.34 & 0.54 & 0.27 & 0.49 & 0.20 & 0.50 & 0.19 & 0.091 & 0.435 \\
\hline $20: 4 n-6$ (ARA) & 0.83 & 0.25 & 0.99 & 0.50 & 0.73 & 0.30 & 0.71 & 0.48 & -0.299 & 0.029 \\
\hline $20: 5 n-3$ (EPA) & 0.14 & 0.09 & 0.16 & 0.13 & 0.17 & 0.12 & 0.16 & 0.08 & 0.176 & 0.158 \\
\hline $22: 4 n-6$ & 0.13 & 0.04 & 0.16 & 0.07 & 0.13 & 0.04 & 0.11 & 0.06 & -0.298 & 0.021 \\
\hline $22: 5 n-3$ (DPA) & 0.17 & 0.09 & 0.20 & 0.13 & 0.16 & 0.09 & 0.15 & 0.09 & -0.237 & 0.042 \\
\hline $22: 5 n-6$ (DPA) & 0.23 & 0.09 & 0.30 & 0.17 & 0.18 & 0.08 & 0.24 & 0.13 & -0.070 & 0.662 \\
\hline $22: 6 n-3$ (DHA) & 0.86 & 0.68 & 1.08 & 0.87 & 0.78 & 0.52 & 0.98 & 0.79 & 0.092 & 0.472 \\
\hline
\end{tabular}

Data are presented as geometric mean and geometric standard deviation (SD). ALA, $\alpha$-linolenic acid; ARA, arachidonic acid; DGLA, dihomo- $\gamma$-linolenic acid; DHA, docosahexaenoic acid; DMA, dimethyl acetal; DPA, docosapentaenoic acid; EPA, eicosapentaenoic acid; GLA, $\gamma$-linolenic acid; LA, linoleic acid.

Table 4 shows that after 21 days of feeding, saturated fatty acid levels in the plasma PL fraction were comparable in both study groups, with the exception of significantly higher 16:0 and significantly lower 18:0 dimethyl acetal (DMA) in the nHMF compared to the cHMF group. 18:1 n-7 was the only monounsaturated fatty acid that was significantly increased in the nHMF group, compared to the cHMF group. Of the PUFAs, 20:3 n-6 (dihomo- $\gamma$-linolenic acid; DGLA), 20:4 n-6 (ARA), 22:4 $n-6,22: 5 n-3$ (n-3 docosapentaenoic acid (n-3 DPA)) levels were significantly lower, whereas 20:5n-3 (eicosapentaenoic acid; EPA) levels were significantly higher in the nHMF compared to the cHMF group. LA, ALA and DHA levels were not significantly different between the two study groups.

In the plasma TAG compartment (Table 5), saturated fatty acid levels did not differ between the two groups after 21 days of dietary intervention. Compared to the cHMF group, the nHMF group showed significant variations in levels of two monounsaturated fatty acids, trans-18.1 and 20:1 n-9. With regard to PUFAs, the nHMF group was characterized by a significant increase in ALA and a significant decrease in ARA, 22:4n-6 and n-3 DPA, when compared to the cHMF group. LA and DHA levels did not differ significantly between the two groups. 
Table 6. Fatty acid profile (g/100 g of fatty acids) of red blood cell phosphatidylcholine (RBC PC) in preterm infants receiving human milk fortified with a control (cHMF) or with a new human milk fortifier (nHMF), before and after 21 days of treatment. Estimations of the treatment effect $\mathrm{nHMF} / \mathrm{cHMF}$ (Difference) and the two-sided $p$-value are given for each fatty acid analysed in the different lipid compartments.

\begin{tabular}{|c|c|c|c|c|c|c|c|c|c|c|}
\hline \multirow{2}{*}{ Fatty Acid } & \multicolumn{4}{|c|}{ cHMF $(n=21)$} & \multicolumn{4}{|c|}{ nHMF $(n=26)$} & \multirow{2}{*}{ Difference } & \multirow{2}{*}{$p$ Value } \\
\hline & Mean & SD & Mean & SD & Mean & SD & Mean & SD & & \\
\hline $14: 0$ & 0.75 & 0.27 & 0.90 & 0.37 & 0.67 & 0.27 & 0.82 & 0.30 & 0.203 & 0.164 \\
\hline $15: 0$ & 0.27 & 0.09 & 0.31 & 0.09 & 0.27 & 0.09 & 0.33 & 0.08 & 0.131 & 0.185 \\
\hline $16: 0$ & 43.91 & 3.45 & 44.01 & 3.36 & 43.87 & 3.91 & 44.23 & 3.34 & 0.035 & 0.165 \\
\hline $16: 1 n-9$ & 0.57 & 0.13 & 0.47 & 0.23 & 0.49 & 0.15 & 0.49 & 0.19 & 0.215 & 0.034 \\
\hline 18:0 & 7.28 & 1.66 & 8.95 & 2.65 & 7.81 & 1.51 & 8.21 & 1.38 & -0.179 & 0.010 \\
\hline 18:0 DMA & 0.10 & 0.04 & 0.08 & 0.03 & 0.09 & 0.03 & 0.07 & 0.03 & -0.065 & 0.636 \\
\hline 18:1 DMA & 0.13 & 0.04 & 0.13 & 0.03 & 0.15 & 0.07 & 0.14 & 0.05 & -0.013 & 0.891 \\
\hline $18: 1 n-7$ & 3.60 & 0.49 & 3.34 & 0.59 & 3.71 & 0.65 & 3.56 & 0.63 & 0.101 & 0.038 \\
\hline $18: 1 n-9$ & 22.74 & 2.11 & 20.89 & 2.49 & 22.19 & 2.63 & 21.86 & 1.94 & 0.068 & 0.038 \\
\hline 18:3 n-6 (GLA) & 0.16 & 0.06 & 0.11 & 0.03 & 0.15 & 0.07 & 0.11 & 0.03 & -0.015 & 0.878 \\
\hline $20: 0$ & 0.10 & 0.05 & 0.11 & 0.05 & 0.10 & 0.04 & 0.11 & 0.04 & 0.061 & 0.673 \\
\hline $20: 1 n-9$ & 0.28 & 0.09 & 0.31 & 0.10 & 0.27 & 0.12 & 0.32 & 0.05 & 0.128 & 0.294 \\
\hline $20: 2 n-6$ & 0.23 & 0.04 & 0.32 & 0.08 & 0.26 & 0.06 & 0.33 & 0.07 & -0.040 & 0.629 \\
\hline 20:3 n-6 (DGLA) & 1.61 & 0.49 & 1.66 & 0.55 & 1.52 & 0.50 & 1.52 & 0.51 & -0.145 & 0.232 \\
\hline $20: 3 n-9$ & 0.48 & 0.35 & 0.39 & 0.24 & 0.37 & 0.22 & 0.41 & 0.25 & -0.124 & 0.536 \\
\hline $20: 4 n-6$ (ARA) & 3.13 & 1.99 & 2.62 & 1.58 & 2.52 & 1.50 & 2.22 & 1.50 & -0.286 & 0.237 \\
\hline $20: 5 n-3$ (EPA) & 0.22 & 0.17 & 0.15 & 0.13 & 0.22 & 0.19 & 0.17 & 0.14 & 0.011 & 0.962 \\
\hline $22: 4 n-6$ & 0.15 & 0.11 & 0.14 & 0.08 & 0.13 & 0.07 & 0.12 & 0.06 & -0.091 & 0.688 \\
\hline $22: 6 n-3$ (DHA) & 0.37 & 0.31 & 0.43 & 0.43 & 0.36 & 0.32 & 0.39 & 0.32 & -0.252 & 0.395 \\
\hline
\end{tabular}

Data are presented as geometric mean and geometric standard deviation (SD). ALA, $\alpha$-linolenic acid; ARA arachidonic acid; DGLA, dihomo- $\gamma$-linolenic acid; DHA, docosahexaenoic acid; DMA, dimethyl acetal; DPA, docosapentaenoic acid; EPA, eicosapentaenoic acid; GLA, $\gamma$-linolenic acid; LA, linoleic acid.

In the RBC-PC compartment, on Day 21 of the study, the nHMF group showed a significant increase in 18:1 n-7, 16:1 n-9 and 18:1 n-9, and a significant decrease in 18:0 levels, compared to the cHMF group (Table 6). No differences were found between groups for the PUFAs.

In the RBC-PE compartment (Table 7), 21 days of dietary intervention with nHMF were associated with a significant decrease in levels of two saturated fatty acids (15:0, 16:0) and a significant increase in levels of two monounsaturated fatty acids (18:1 n-7, 20:1 n-9), when compared to the cHMF intervention. A significant increase in levels of several PUFAs, DGLA, EPA, $n-3$ DPA, and DHA was also observed in the nHMF group. No differences in ARA levels were observed between the two groups. 
Table 7. Fatty acid profile (g/100 g of fatty acids) of red blood cell phosphatidylethanolamine (RBC $\mathrm{PE}$ ) in preterm infants receiving human milk fortified with a control (cHMF) or with a new human milk fortifier (nHMF), before and after 21 days of treatment. Estimations of the treatment effect $\mathrm{nHMF} / \mathrm{cHMF}$ (Difference) and the two-sided $p$-value are given for each fatty acid analysed in the different lipid compartments.

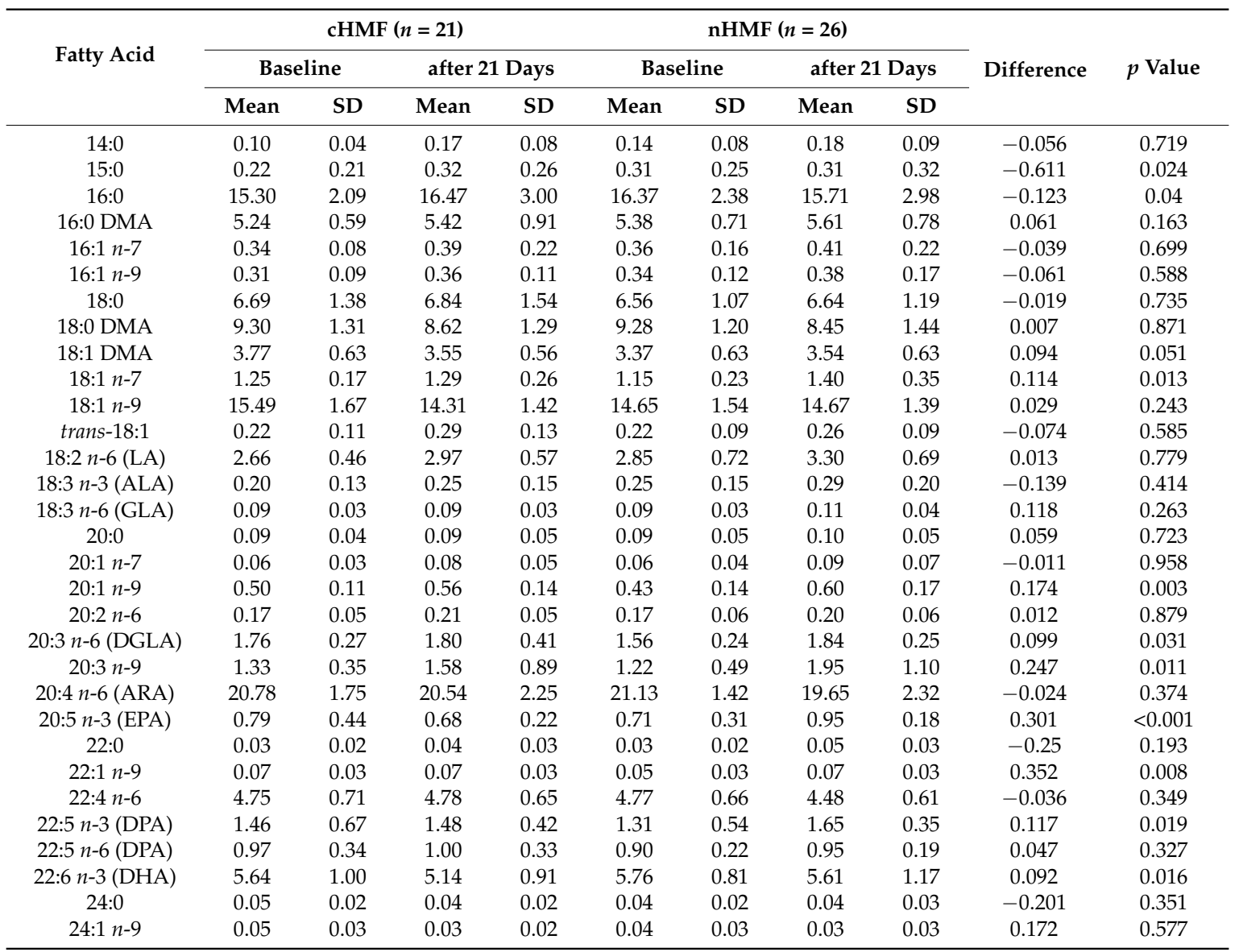

Data are presented as geometric mean and geometric standard deviation (SD). ALA, $\alpha$-linolenic acid; ARA, arachidonic acid; DGLA, dihomo- $\gamma$-linolenic acid; DHA, docosahexaenoic acid; DMA, dimethyl acetal; DPA, docosapentaenoic acid; EPA, eicosapentaenoic acid; GLA, $\gamma$-linolenic acid; LA, linoleic acid.

\section{Discussion}

The lipid composition of the nHMF is intended to provide significant quantities of dietary MCFA 8:0 and 10:0, ALA and DHA, as a complement to HM innate lipid content (Table 2). In the present study, a subset of HM samples was fortified with nHMF to validate both its addition rate and composition in accordance to the current recommendations (Table 3). It is important to note that large inter-individual variation of ARA and DHA content in HM has been observed mainly due to the influence of maternal diet [7]. In the present study, comparison of the fatty acid profile of erythrocyte and plasma lipids in infants fed HM fortified with nHMF or cHMF provides valuable insight into the fatty acid metabolism of preterm infants. Based on the full dataset, Figure 1 gives a comprehensive representation of the changes in fatty acid levels when preterm infants are fed HM fortified with the nHMF compared to preterm infants fed HM fortified with cHMF. Figure 1 also provides a putative explanation of the factors driving these changes. Almost all the fatty acids of the $n-7, n-9, n-6$ and $n-3$ series were impacted by the nHMF, thus confirming a highly active fatty acid metabolism in the preterm infants of our study (Figure 1). In the present study, an accumulation of metabolites formed by $\Delta 9$-desaturation and elongation products of saturated fatty acids, up to 22:1 n-9 in RBC-PE, is observed (Figure 1). Increased levels of elongation and desaturation metabolites formed from saturated fatty acids (e.g., palmitic 
and stearic acids) have been previously observed in rats fed with MCT and labelled palmitic and stearic acid [16]. In this animal experiment, Leveille et al. demonstrated that feeding 8:0 and 10:0 acids stimulates the elongation of exogenous palmitic acid as well as formation of oleic acid from stearic acid by $\Delta 9$-desaturation [16]. It can therefore be hypothesized that feeding MCFA provided by dietary MCT stimulates the metabolism of saturated fatty acids provided by human milk as represented schematically in Figure 1. Monounsaturated fatty acids are important components of polar lipids in the central nervous system and in particular in the myelin sheath $[17,18]$. Myelination begins during the last trimester of gestation, and nutrition in preterm infants plays a critical role in this process [19]. One can therefore hypothesize that supplying dietary lipids to stimulate the formation of monounsaturated fatty acids, and in particular $n-9$ long-chain monounsaturated fatty acids, during the neonatal period is an appealing nutritional strategy to support the myelination process in the preterm infant.

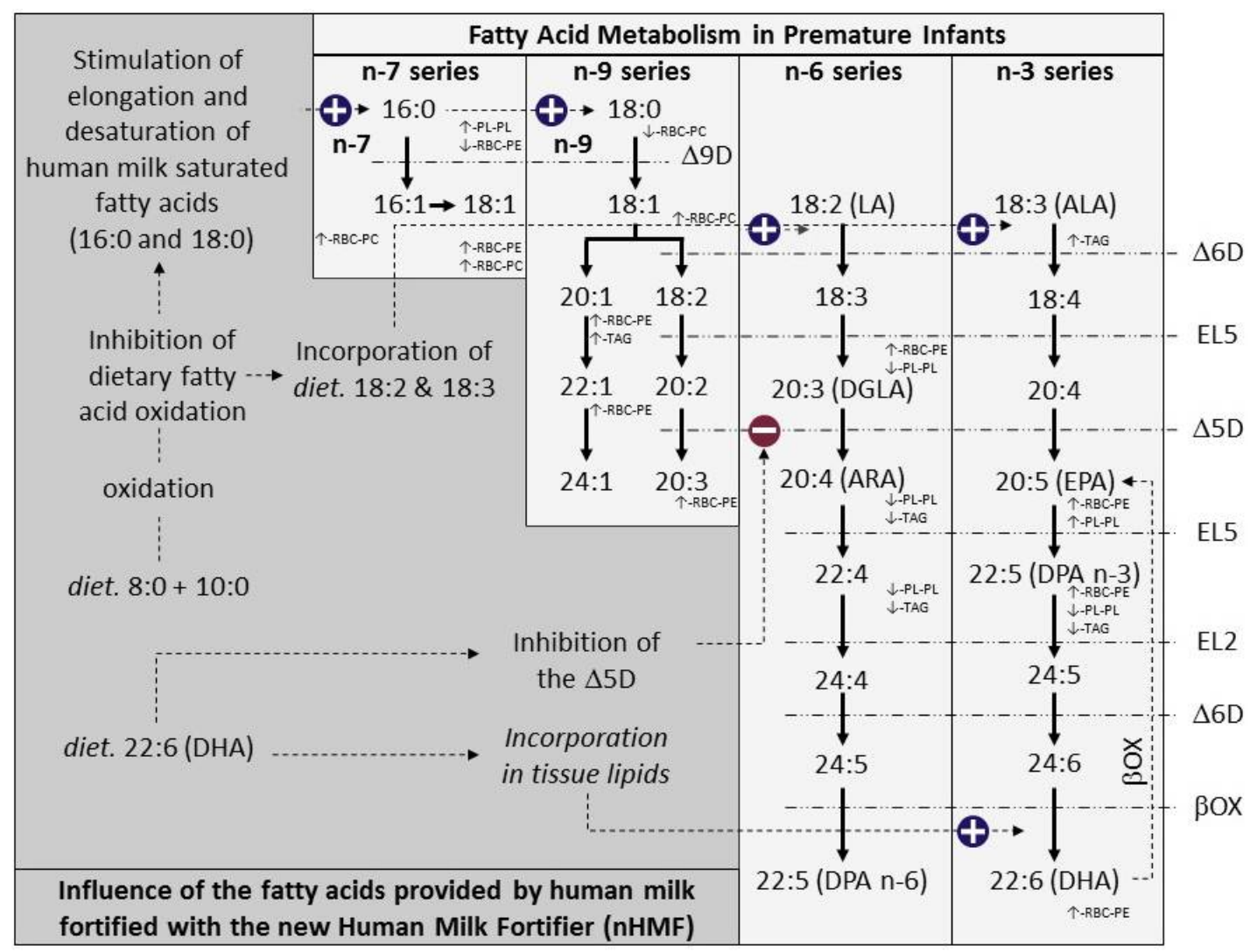

Figure 1. Schematic representation of the influence of dietary fatty acids provided by the nHMF. Differences (shown by the $\uparrow$ and $\downarrow$ symbols) between relative concentrations of FA provided by the nHMF or the $\mathrm{cHMF}$ in the compartments assessed are statistically significant $(p<0.05)$. Metabolic pathways responsible for the biosynthesis of FA from the $n-3, n-6, n-7$, and $n-9$ series are displayed together with the enzymes involved $(\Delta 9 \mathrm{D}, \Delta 9$-desaturase; $\Delta 6 \mathrm{D}, \Delta 6$-desaturase, EL5, elongase $5 ; \Delta 5 \mathrm{D}$, $\Delta 5$-desaturase; EL2, elongase 2; $\beta \mathrm{OX}, \beta$-oxidation).

The use of essential fatty acids, and ALA in particular, in the composition of the nHMF led to an increased incorporation of ALA in plasma TAG of pre-term infants fed nHMF (Table 5 and Figure 1). Enhanced ALA intake (Table 2) led to greater accumulation of ALA metabolites (including EPA and $n-3$ DPA) formed by elongation and desaturation, and notably in RBC-PE (Table 7 and Figure 1). Dietary DHA provided by the nHMF led to beneficial increases in DHA levels in RBC-PE, which has been used as a proxy for neural tissue lipids [20]. LA levels were almost identical in nHMF and cHMF when mixed with HM (Table 2). Levels of LA and its fatty acid metabolites were therefore not enriched in 
the blood lipids of pre-term infants, with the one exception of higher levels of DGLA in the RBC-PE of infants fed nHMF (Figure 1). Slightly lower levels of LA metabolites, and DGLA, ARA and $n-3$ DPA in particular, were observed in plasma lipids, suggesting potential inhibition of LA metabolism by the pre-formed DHA provided to pre-term infants fed nHMF. However, such modulation of the $n-6$ fatty acids by dietary DHA was not observed in RBC-PE (Table 7). One may therefore hypothesize that the lower levels of $n-6$ fatty acids observed in plasma lipids do not impact the fatty acid composition of tissue lipids.

One important limitation of the present study is the limited duration of the feeding period (21 days). Therefore, the observed impact of nHMF on the composition of circulatory lipids and in particular on the RBC membrane lipids should be critically considered. It is known indeed, that RBC have a half-life longer than the observation period. Pre-term infants are also developing rapidly, and therefore it is difficult to predict the impact of the nHMF on circulatory lipids over a longer time of exposure.

\section{Conclusions}

The present study shows that fortifying human milk with a new fortifier containing MCT, as a source of MCFA, ALA and DHA led to an increased incorporation of $n-9$ long-chain monounsaturated fatty acids in RBC-PE, which is a reliable indicator of fatty acid metabolism in premature infants.

Author Contributions: J.R. and J.-C.P. designed the study with input from C.B. and P.S.; C.B., J.R., J.-C.P., and E.S. conducted the study. C.B.-V., L.C., and C.C.-H. conducted the sample analysis. J.J. conducted the data analysis. C.B., C.B.-V., L.C., P.S., J.J., C.C.-H., L.A., J.R., J.-C.P., E.S., N.P.H., and F.D. interpreted the data and critically reviewed the paper. F.D. wrote the paper, and F.D. and N.P.H. revised the paper.

Funding: This study was sponsored by Nestlé Nutrition.

Acknowledgments: The authors thank the families of the infants who participated in the study, as well as the research staff at each participating institution. We also thank Christelle Perdrieu and Samir Dahbane from the Clinical Development Unit at the Nestlé Research Center for assistance with trial management and Christele Closse/COPEDA who provided medical writing services on behalf of Nestlé Nutrition. Portions of these data were presented in abstract form at the World Congress of Pediatric Gastroenterology, Hepatology, and Nutrition, Montreal, Canada, 5-8 October 2016.

Conflicts of Interest: P.S., J.J., C.C.-H., L.A., N.P.H., and F.D. are employees of Nestlé SA. C.B., J.R., J.-C.P., and E.S. received research funding from Nestlé Nutrition. J.R., J.-C.P., and C.B. are consultants for Nestlé Nutrition. C.B.-V. and L.C. have no conflicts of interest to declare.

\section{References}

1. Harris, H.; Baack, M. Beyond building better brains: Bridging the docosahexaenoic acid (DHA) gap of prematurity. J. Perinatol. 2015, 35, 1-7. [CrossRef] [PubMed]

2. Martin, C.R. Fatty acid requirements in preterm infants and their role in health and disease. Clin. Perinatol. 2014, 41, 363-382. [CrossRef] [PubMed]

3. Innis, S.M. Essential fatty acid transfer and fetal development. Placenta 2005, 26, S70-S75. [CrossRef] [PubMed]

4. Baack, M.L.; Puumala, S.E.; Messier, S.E.; Pritchett, D.K.; Harris, W.S. What is the relationship between gestational age and docosahexaenoic acid (DHA) and arachidonic acid (ARA) levels? Prostaglandins Leukot. Essent. Fatty Acids 2015, 100, 5-11. [CrossRef] [PubMed]

5. Lapillonne, A.; Groh-Wargo, S.; Lozano Gonzalez, C.H.; Uauy, R. Lipid needs of preterm infants: Updated recommendations. J. Pediatr. 2013, 162, S37-S47. [CrossRef] [PubMed]

6. Lapillonne, A. Enteral and parenteral lipid requirements of preterm infants. World Rev. Nutr. Diet. 2014, 110, 82-98. [PubMed]

7. Brenna, J.T.; Varamini, B.; Jensen, R.G.; Diersen-Schade, D.A.; Boettcher, J.A.; Arterburn, L.M. Docosahexaenoic and arachidonic acid concentrations in human breast milk worldwide. Am. J. Clin. Nutr. 2007, 85, 1457-1464. [CrossRef] [PubMed]

8. Billeaud, C.; Bouglé, D.; Sarda, P.; Combe, N.; Mazette, S.; Babin, F.; Entressangles, B.; Descomps, B.; Nouvelot, A.; Mendy, F. Effects of preterm infant formula supplementation with $\alpha$-linolenic acid with a 
linoleate/ $\alpha$-linolenate ratio of 6: A multicentric study. Eur. J. Clin. Nutr. 1997, 51, 520-526. [CrossRef] [PubMed]

9. Rigo, J.; Hascoët, J.-M.; Billeaud, C.; Picaud, J.C.; Mosca, F.; Rubio, A.; Saliba, E.; Radkë, M.; Simeoni, U.; Guillois, B.; et al. Growth and nutritional biomarkers of preterm infants fed a new powdered human milk fortifier: A randomized trial. J. Pediatr. Gastroenterol. Nutr. 2017, 65, e83-e93. [CrossRef] [PubMed]

10. Cruz-Hernandez, C.; Goeuriot, S.; Giuffrida, F.; Thakkar, S.K.; Destaillats, F. Direct quantification of fatty acids in human milk by gas chromatography. J. Chromatogr. A 2013, 1284, 174-179. [CrossRef] [PubMed]

11. Folch, J.; Lees, M.; Sloane Stanley, G.H. A simple method for the isolation and purification of total lipids from animal tissues. J. Biol. Chem. 1957, 226, 497-509. [PubMed]

12. Peuchant, E.; Wolff, R.; Salles, C.; Jensen, R. One-step extraction of human erythrocyte lipids allowing rapid determination of fatty acid composition. Anal. Biochem. 1989, 181, 341-344. [CrossRef]

13. Morrison, W.R.; Smith, L.M. Preparation of fatty acid methyl esters and dimethylacetals from lipids with boron fluoride-methanol. J. Lipid. Res. 1964, 5, 600-608. [PubMed]

14. Koletzko, B.; Poindexter, B.; Uauy, R. Recommended nutrient intake levels for stable, fully enterally fed very low birth weight infants. In Nutritional Care of Preterm Infants: Scientific Basis and Practical Guidelines; Koletzko, B., Poindexter, B., Uauy, R., Eds.; Karger: Basel, Switzerland, 2014; pp. 297-299.

15. Agostoni, C.; Buonocore, G.; Carnielli, V.P.; de Curtis, M.; Darmaun, D.; Desci, T.; Domellöf, M.; Embleton, N.D.; Fusch, C.; Genzel-Boroviczeny, O.; et al. Enteral nutrient supply for preterm infants: Commentary from the European Society for Paediatric Gastroenterology, Hepatology, and Nutrition Committee on Nutrition. J. Pediatr. Gastroenterol. Nutr. 2010, 50, 85-91. [CrossRef] [PubMed]

16. Leveille, G.A.; Pardini, R.S.; Tillotson, J.A. Influence of medium-chain triglycerides on lipid metabolism in the rat. Lipids 1967, 4, 287-294. [CrossRef] [PubMed]

17. Svennerholm, L.; Vanier, M.T. Lipid and fatty acid composition of human cerebral myelin during development. Adv. Exp. Med. Biol. 1978, 100, 27-41.

18. O'Brien, S.J.; Sampson, E.L. Lipid composition of the normal human brain: Gray matter, white matter, and myelin. J. Lipid Res. 1965, 6, 537-544. [PubMed]

19. Quarles, R.H.; Macklin, W.B.; Morell, P. Myelin formation, structure and biochemistry. In Basic Neurochemistry: Molecular, Cellular and Medical Aspects, 7th ed.; Brady, S., Siegel, G., Albers, R.W., Price, D., Eds.; Academic Press: San Diego, CA, USA, 2005; pp. 51-71.

20. Innis, S.M. Human milk and formula fatty acids. J. Pediatr. 1992, 4, S56-S61. [CrossRef] 